\title{
Editorial
}

\section{Separation surgery}

\author{
Charles Fisher M.D., M.H.Sc., and Juliet BatKe, B.Sc.
}

\begin{abstract}
Department of Orthopaedics, University of British Columbia, Combined Neurosurgical and Orthopaedic Spine Program, Vancouver General Hospital, Vancouver, British Columbia, Canada
\end{abstract}

The management of patients with spine metastases from a functional and health-related quality of life (HRQOL) perspective is one of the most challenging issues faced by both oncologists and spine surgeons. Higher levels of evidence have been achieved over the past decade in defining the positive impact of surgery and/or conventional external beam radiation therapy (cEBRT) in these patients. ${ }^{1,3}$ More recently, stereotactic radiosurgery (SRS) has provided a successful treatment option for traditionally radioresistant tumors, but has been limited in a setting where extensive epidural disease restricts radiation dose due to the threat of cord toxicity. ${ }^{2}$ In this patient group, maximally invasive surgery to achieve gross-total resection of the tumor was often necessary to prevent local recurrence and optimize HRQOL. This surgery is resource intensive, with a not-insignificant adverse event rate, so it is not ideal in those patients who are systemically compromised and have limited life expectancy. To address this problem, Laufer et $a .^{4}$ report on a treatment method of surgically removing the epidural disease (separation surgery) and then administering high-dose SRS to the remaining tumor, thus providing a means of potentially achieving local control while minimizing surgical insult and cord toxicity.

The study has a strong rationale and clear purpose: to determine local recurrence rate after separation surgery and SRS. The study design is a retrospective case series involving a relatively large consecutive patient cohort treated at a specialized cancer center between 2002 and 2011. The study design is reasonable for the research question, given the complexity of the patient population and the evolution of the treatment method, but not optimal with the number of potential confounders, treatment variability, and difficulty in accurately determining the primary or dependent outcome. Unfortunately, the validity or accuracy of local recurrence determination is difficult due to changes in technology over time, reviewer variability (imaging reports were used as well as neuro- radiology and neurosurgeon review), lack of technique standardization (CT myelogram and MRI), effect of spinal implant imaging artifact, lack of standardized time periods, and quantitative evaluation. With these limitations, the magnitude of the group without local recurrence ( $82 \%$ of the patients) is questionable; however, such an impressive effect change cannot be ignored, and the positive effect is probably a true finding.

The authors use a very appropriate and robust statistical analysis to try and overcome the treatment variability and aforementioned limitations. The variability leaves the study underpowered on a number of secondary outcomes, but trends can be observed. The study does not include a patient-focused HRQOL instrument to ensure that impressive imaging outcomes are correlated with those of the patient. This omission is not critical, given the research question being asked, and using local recurrence rate as the primary outcome is a reasonable surrogate for HROQL outcome in prospective tumor research. Finally, the generalizability of the study's findings must be considered. The breadth and variability of the cohort supports external validity, but the specialization and technology of the center and subspecialization of the surgeons and oncologists may limit it.

The management of patients with symptomatic spinal metastases is difficult. Carrying out high-quality clinical research involving patients whose quality of life you are trying to optimize for their remaining time is even more demanding. The authors should be congratulated for giving us evidence for a treatment method that potentially fills a therapeutic void in this truly deserving patient population. Can we implement the findings from this study into our clinical practice despite its being at the lower level of study design hierarchy? Though the study is statistically sound and optimal within the restraints of a retrospective design, its limitations diminish its impact. The effect size, external validity within the context of large cancer centers, and the unique patient population probably support cautious implementation of this treatment method. As the technique evolves and is used by more centers, prospective evaluation will be essential. (http://thejns.org/doi/abs/10.3171/2012.10.SPINE12743)

\section{Disclosure}

The authors report no conflict of interest. 


\section{References}

1. Bilsky MH, Laufer I, Burch S: Shifting paradigms in the treatment of metastatic spine disease. Spine (Phila Pa 1976) 34 (22 Suppl):S101-S107, 2009

2. Gerszten PC, Burton SA, Ozhasoglu C, Welch WC: Radiosurgery for spinal metastases: clinical experience in 500 cases from a single institution. Spine (Phila Pa 1976) 32:193-199, 2007

3. Gerszten PC, Mendel E, Yamada Y: Radiotherapy and radiosurgery for metastatic spine disease: what are the options, indications, and outcomes? Spine (Phila Pa 1976) 34 (22 Suppl): S78-S92, 2009

4. Laufer I, Iorgulescu JB, Chapman T, Lis E, Shi W, Zhang Z, et al: Local disease control for spinal metastases following "separation surgery" and adjuvant hypofractionated or high-dose single-fraction stereotactic radiosurgery: outcome analysis in 186 patients. Clinical article. J Neurosurg Spine [epub ahead of print January 22, 2013. DOI: 10.3171/2012.11.SPINE12111]

\section{Response}

MARK H. BILSKY, M.D.

Department of Neurosurgery and Spine Tumor Center, Memorial Sloan-Kettering Cancer Center, and Department of Neurological Surgery, Weill Cornell Medical College, New York, New York

We would like to thank Dr. Fisher and Ms. Batke for their insightful and thoughtful review of our paper (Local disease control for spinal metastases following "separation surgery" and adjuvant hypofractionated or high-dose single-fraction stereotactic radiation therapy: outcome analysis in 186 patients). The holy grail in metastatic spine tumor surgery has been to minimize the extent of surgical interventions while maximizing tumor control in order to improve quality of life and return patients expeditiously to systemic therapy. The fundamental goals of surgery are decompression of the spinal cord to maintain or regain neurological function and placement of instrumentation to stabilize the spine. In our experience, tumor recurrence is predicated not on the extent of resection, but on the tumor biology, and, thus, the responsiveness to radiation therapy. One of the most difficult scenarios for a spine tumor surgeon is to perform an extensive anterior and posterior operation to resect gross tumor only to face a recurrence 3 months after surgery. The introduction of SRS as a postoperative adjuvant is beginning to shift treatment paradigms to less-aggressive surgical approaches because the tumor responses are histologyindependent and durable, unlike responses to cEBRT. Separation surgery limits tumor resection to the epidural space in order to reconstitute the spinal fluid space around the spinal cord and facilitate delivery of an effective SRS dose that remains within the constraints of spi- nal cord toxicity. The tumor control provided by adjuvant SRS even in the setting of large and previously irradiated tumors allows surgeons to decrease the extent, morbidity, and length of surgery.

The authors correctly note that "local recurrence determination is difficult" to assess based on a number of parameters, and this represents a limitation in our study, which examines tumor recurrence as the primary endpoint. Standardization of postoperative imaging is difficult in metastatic cancer patients, who often have other competing problems that become priorities in their care. Nevertheless, all patients received baseline postoperative myelogram/CT studies for SRS simulation and the majority also had pre-SRS MRI studies. Both CT/myelogram and MRI are very sensitive for detecting progression of epidural, bone, and paraspinal tumor even in the presence of titanium hardware. Additionally, all patients reported on in our study uniformly had post-SRS imaging every 3-6 months unless contraindicated by extensive progression of systemic disease. All available studies were reviewed by a single neuroradiologist who was blinded to the dose. Ultimately, better radiological assessments will be needed to delineate not only tumor recurrence or progression, but actual response to SRS. Recent evidence from our institution suggests that dynamic contrast-enhanced MR images predict tumor response to therapy (unpublished data); however, it remains to be determined whether these will be predictive in the presence of titanium implants.

Perhaps the greatest barrier to widespread adoption of the new treatment paradigm of separation surgery and SRS is the availability of radiation delivery systems. Fortunately, the required technology is becoming widely available outside of large academic cancer centers as the recognition of its utility becomes clear, not only in brain and spine applications, but also in the lung, pancreas, and prostate. Ultimately, the issue may not be whether this technology is available in a given institution, but rather development and adoption of standards for the delineation of tumor contours and dosing schedules that will optimize tumor control following separation surgery. In our study, higher-dose single-fraction or hypofractionated schedules demonstrated improved control rates compared to low-dose hypofractionated radiation treatment independent of tumor histology and prior radiation therapy. Our hope is that studies such as ours will provide the groundwork necessary for the development of guidelines for postoperative SRS in order to meet the goals of therapy with reduced morbidity in this complicated patient population.

Please include this information when citing this paper: published online January 22, 2013; DOI: 10.3171/2012.10.SPINE12743. 